\title{
Interdisziplinäre Führungsstrukturen zur Lösung von Koordinationsproblemen
}

\author{
Eine Organisationsentwicklung sollte in der Klinik für Innere Medizin des Univer- \\ sitätsspitals Basel das Zusammenspiel zwischen den unterschiedlichen Funktions- \\ bereichen und Hierarchieebenen verbessern. Aufgaben, Verantwortung und Kompe- \\ tenzen von Ärzten und Pflegenden wurden in einer interdisziplinären Führungs- \\ struktur geregelt.
}

Germaine Ezea,

Jürg Leuppi ${ }^{b}$, Claude Rosselet ${ }^{c}$

a Ehem. Fachbereichsleiterin Pflege und MTT Innere Medizin am Universitätsspital Basel

b Prof. Dr., Stellvertretender Chefarzt Innere Medizin am Universitätsspital Basel

c lic. oec. HSG, Inscena Systemische Beratung $\mathrm{GmbH}$ Männedorf
Korrespondenz:

lic. oec. Claude Rosselet Inscena Systemische Beratung $\mathrm{GmbH}$

Alte Landstrasse 161B

CH-8708 Männedorf

Tel. 0449206010

c.rosselet[at]inscena.ch
Der prüfende Blick auf die Leistungserbringung von Institutionen des Gesundheitswesens bringt zwangsläufig die Forderung nach Rationalisierung mit sich. Oft genug wird bei der Suche nach entsprechendem Potential allerdings vergessen, dass es sich bei diesen Institutionen nicht um Betriebe klassischen $\mathrm{Zu}$ schnitts handelt, sondern um sogenannte «Professional Organizations». Bereits 1989 wies der kanadische Professor Henry Mintzberg auf die Besonderheiten dieses Organisationstypus hin [1]: Die Erfüllung der Primäraufgabe erfordert den Einsatz gründlich ausgebildeter Spezialisten, die sich vorab an den Patienten bzw. Klienten orientieren. Die Leistung kann zudem nicht mit den üblichen betriebswirtschaftlichen Verfahren gemessen und standardisiert werden, denn sie beruht auf einem ausgeklügelten, sich am Einzelfall orientierenden (Kunst-)Handwerk. Neben Spitälern werden diesem Organisationstyp zugerechnet: Universitäten, Gerichte, Treuhand- und Beratungsfirmen.

Dieser Organisationstyp hat neben klaren Vorzügen - wie z. B. ein hohes Arbeitsethos und eine ausgeprägte intrinsische Motivation der Mitarbeitenden eine Reihe von Mängeln: die schwierige Koordination zwischen den Professionals und zwischen Professionals und den Mitarbeitenden der unterstützenden Organisationseinheiten, die beinahe ausschliessliche Loyalität der Professionals gegenüber ihrem Beruf und weitgehend fehlende, auf induktivem bzw. divergentem Denken beruhende grundlegende Innovationskraft.

Es wäre kurzsichtig, diesen Problemen mit Managementpraktiken zu Leibe rücken zu wollen, wie sie in der Industrie oder bei Banken und Versicherungen üblich sind. Die operationellen Funktionen von Institutionen des Gesundheitswesens lassen sich nicht einfach «industrialisieren», denn die Logik der hier geltenden Routinen entspringt dem Geist eines professionellen State of the Art. Somit sind die Arbeitsprozesse gegenüber der Standardisierung eines Taylorschen Zuschnitts einigermassen immun.

Erfolg hat eher, wer flexible und interdisziplinäre Teams zur Lösung gemeinsamer Probleme einsetzt,

\section{Les structures de direction inter-} disciplinaires aident à résoudre les problèmes de coordination

Si l'on jette un regard critique sur les institutions du domaine de la santé, on en vient immanquablement à la rationalisation. Pourtant, lorsqu'on recherche des solutions, on oublie fréquemment que ces institutions n'ont pas le profil d'entreprises classiques mais celui $d^{\prime}$ '«organisations professionnelles». Tout le pan opérationnel des institutions du domaine de la santé ne se laisse pas simplement «industrialiser». ๔uvrer de manière flexible et interdisciplinaire à la recherche de solutions communes, instaurer une structure de direction concertée autour de réunions sur des sujets clairement définis et développer une culture collective basée sur des normes et des valeurs contraignantes sont en revanche source de succès.

Dans le présent article, vous apprendrez comment le service de médecine interne de l'hôpital universitaire de Bâle a pu, en introduisant un système de direction interdisciplinaire, aplanir les paradoxes et améliorer la coordination entre les différents protagonistes. L'hôpital a en effet mis sur pied une équipe dirigeante interdisciplinaire pour son unité de lits, dans laquelle le médecin-chef, son suppléant, la responsable de l'unité de soins et les représentants de l'administration organisent régulièrement des réunions sur des sujets concernant l'unité de lits.

eine Führungsstruktur mit aufeinander abgestimmten Besprechungen zu klar definierten Themen implementiert und auf die Entwicklung einer gemein- 
samen Kultur auf der Basis verbindlicher Normen und Werte baut.

Dieser Beitrag beschreibt, wie in der «Inneren Medizin» des Universitätsspitals Basel (USB) durch die Einführung einer interdisziplinären Führungsstruktur grundlegende Paradoxien entschärft und die Koordination zwischen den Akteuren verbessert werden konnte.

\section{OE-Prozess zur Entwicklung einer interdisziplinären Führungsstruktur} Eine Organisationsentwicklung (OE) sollte in der Klinik für Innere Medizin des Universitätsspitals Basel das Zusammenspiel zwischen den unterschiedlichen Funktionsbereichen und Hierarchieebenen verbessern. Dabei waren insbesondere die Aufgaben, Verantwortung und Kompetenzen der beteiligten Funktionsträger im Rahmen einer interdisziplinären Führungsstruktur zu regeln.

\section{Grundlegende Paradoxien als implizite Spannungsmomente}

Nicht der mangelnde gute Wille der einzelnen Akteure macht gelingende Zusammenarbeit bisweilen zu einer schier unlösbaren Sache. Es sind eher die von unterschiedlichen und sich gelegentlich sprunghaft ändernden Erwartungen aus dem organisationalen Kontext - Patienten, Angehörige, Berufsgruppen, Politiker, Universität u.a.m. - hervorgerufenen und am Leben gehaltenen Paradoxien.

Diese Paradoxien bilden einen permanenten Unruheherd. Häufig treten sie nicht deutlich ins
- Hierarchiedenken versus Orientierung an betrieblichen Notwendigkeiten und Sachthemen.

\section{Funktionale Ausdifferenzierung versus} Zusammenspiel auf den Bettenstationen

Die primäre Organisationsstruktur der «Inneren Medizin» basiert auf der Ausdifferenzierung in die Professionswelten der Ärzteschaft, Pflege und Administration. Jede dieser Welten folgt ihrer eigenen Logik. Hinter ihren ganz spezifischen Routinen und Praktiken bildet die Professionswelt einen eigenen Horizont, bestehend aus identitätstiftenden Erklärungen, aus. Vor diesem Horizont macht die Handlung der einzelnen Akteure jeweils ihren Sinn - und erfährt dadurch ihre Legitimation.

Das sukzessiv erfolgte Auseinanderdriften der Professionswelten fand in Folgendem seinen Niederschlag:

- Rückzug auf die «eigene» Position bei schwierigen Abstimmungsprozessen und - in der Folge Durchsetzung von suboptimalen Lösungen aus der je ganz spezifischen Interessenlage heraus;

- Negative Zuschreibungen nach dem Schema: «Bei uns funktioniert alles gut, die Probleme entstehen, weil die anderen es falsch machen.»

- Gegenseitige Abschottung auf kollektiver und Resignation auf individueller Ebene infolge ungelöster Konflikte.

Dies behinderte das Zusammenspiel zum Wohle des Patienten. Und dies sowohl an seinem Bett als auch im Umfeld seines Bettes. Denn dazu bedarf es ver-

\section{Das Rotationsprinzip im ärztlichen Einsatz führte bei der Pflege zum Eindruck, die Ärzte kümmerten sich zu wenig um das Funktionieren des Betriebes auf der Station.}

Bewusstsein und finden in Form von Unmut, Frustration und Resignation ihren Ausdruck. Die durch Paradoxien ausgelösten Spannungen können nicht vom einzelnen Mitarbeiter allein, sondern nur gemeinsam durch zweckmässige und sachorientierte Kommunikation im Rahmen einer robusten Führungsstruktur «gemanagt» (aber nie «endgültig» gelöst) werden.

Im Verlaufe von Interviews, die mit leitenden Ärzten und Pflegenden durchgeführt wurden, konnten drei zentrale Paradoxien ausgemacht werden:

- Funktionale Ausdifferenzierung des Bereichs Innere Medizin in Ärzte, Pflege und Administration versus interdisziplinäres Zusammenwirken in den Bettenstationen;

- Ausbildungsstätte versus nach betriebswirtschaftlichen Gesichtspunkten durchrationalisierter Betrieb mit hohem Qualitätsanspruch und geringer Fehlertoleranz; bindlicher Absprachen zwischen den Mitgliedern der verschiedenen Professionswelten zu folgenden Aspekten:

- Was braucht ein Patient an Behandlung und an Pflege (fachlicher Aspekt)?

- Wie sollen Behandlung und Pflege im Einzelfall aufeinander abgestimmt und organisatorisch geregelt werden (organisationaler Aspekt)?

\section{Ausbildungsstätte versus robuster Betrieb}

Die Aus- und Weiterbildung hat in der «Inneren Medizin» einen hohen Stellenwert. Allerdings liegt der Akzent eher auf der Qualifizierung von Assistenz- und Oberärzten. Ärzte verweilen relativ kurze Zeit auf einer Station, was unter dem Aspekt der Personenqualifizierung Sinn macht: Unterschiedliche Kontexte ermöglichen vielerlei Erfahrungen und entsprechend Vermehrung des Wissens. Deshalb wird das 


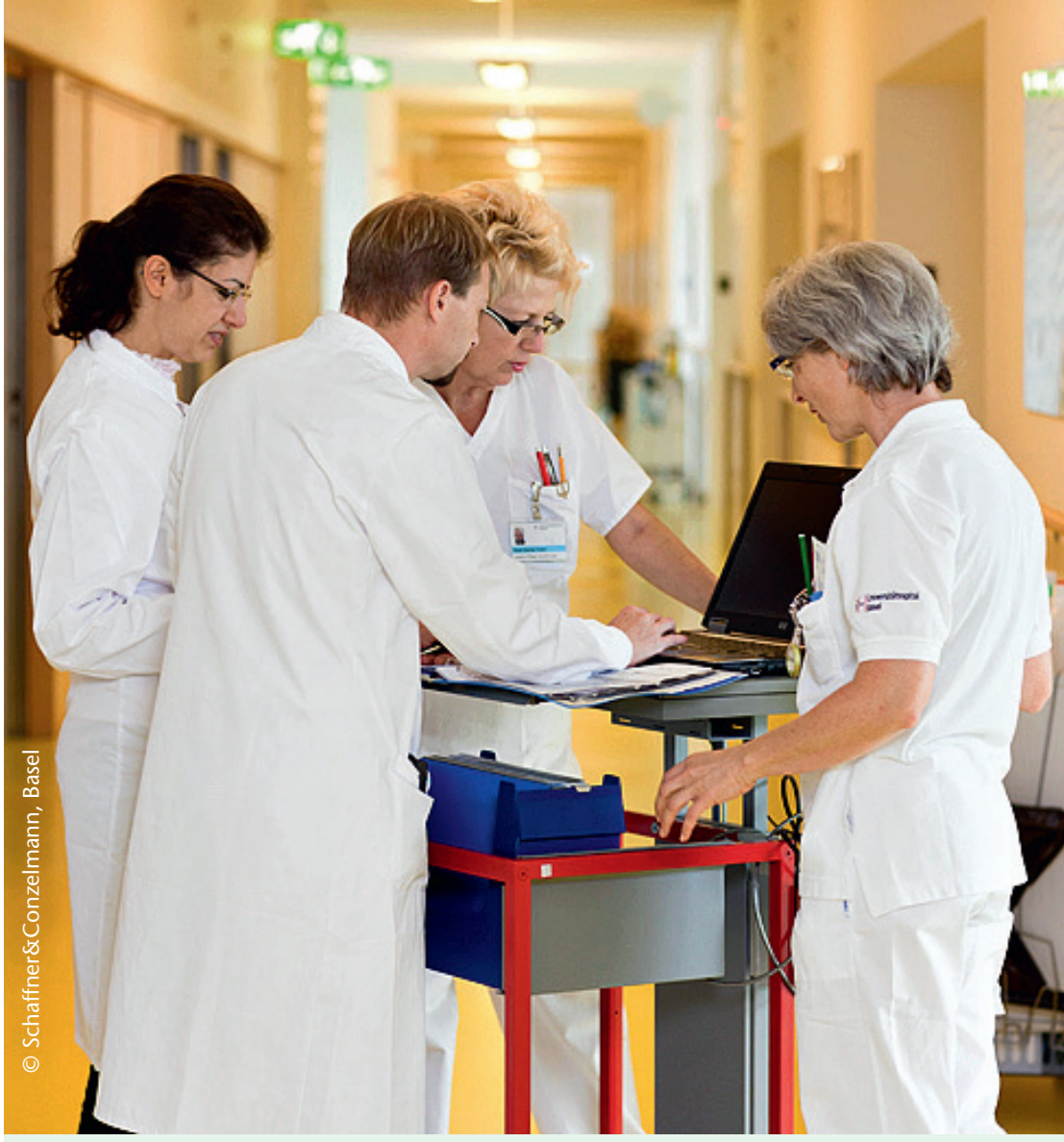

Erfolg hat, wer flexible und interdisziplinäre Teams zur Lösung gemeinsamer Probleme einsetzt.
Die Leitdifferenz «Oben/Unten» schien im Bewusstsein sämtlicher Mitarbeitenden tief verankert zu sein. Allerdings sei an dieser Stelle gleich angemerkt, dass «Unten» nicht gleichbedeutend mit machtlos und «Oben» mit mächtig war. Tatsächlich widerspiegelte sich die Machtfrage in vielen Sachthemen. Diese Tendenz wurde durch die primäre Organisationsstruktur noch unterstützt: Diese differenziert, wie bereits erwähnt, Berufsgruppen aus. Letztere geniessen unterschiedliches Prestige. Die Identifikation mit der gemeinsam erbrachten Leistung rückte dabei gelegentlich etwas in den Hintergrund.

\section{Fazit}

Dass Organisation nicht ein für allemal geregelt und dann «vergessen» werden kann, hat seinen Grund in Paradoxien. Diese generieren einen permanenten Abstimmungsbedarf, der in unterschiedlichsten Kommunikationsräumen (Workshops, Sitzungen, Korridor- und Kantinengesprächen) zwischen den beteiligten Akteuren abgearbeitet werden muss. Der funktionale Ort, an dem dies geschehen kann, sind Besprechungen. Doch: Welche Besprechungen bringen tatsächlich einen Nutzen? Welche Themen gibt es dort? Und wer wird dazu eingeladen?

\section{Lösungsansatz: Führungsstruktur basierend auf Durchsprachen zwischen den Funktionsbereichen}

Der Grossteil der auf Behandlung und Pflege fokussierten Prozesse findet innerhalb der einzelnen Bettenstationen der Klinik für Innere Medizin statt. Dort haben die Akteure zusammenzuspielen, die klinikweit in drei Disziplinen ausdifferenziert sind. Besprechungen zwischen Ärzten und Pflegenden gab es zwar immer schon zu Fragen der Behandlung und Betreuung einzelner Patienten. In diesem anlassbezogenen Rahmen konnten auch organisatorische Fragen thematisiert werden. Im Hinblick auf Fragen der übergreifenden, interdisziplinären Steuerung der Organisationseinheit «Bettenstation» gab es hingegen keine strukturell verankerten Möglichkeit von Besprechungen zwischen Ärzten und Pflegenden.

Ausser auf der Ebene der Klinikleitung waren Sitzungen denn auch nur innerhalb der einzelnen Professionswelten strukturell verankert: In der Regel zu intradisziplinären Sachfragen, seltener zu Fragen der Betriebsführung und -organisation. Für die Bearbeitung solcher Fragen wurden bei Bedarf Arbeitsgruppen eingesetzt. Sie entwickelten in Bezug auf gerade anstehende Probleme Lösungen, deren Umsetzung allerdings nicht durch eine auf die einzelne Bettenstation durchgreifende und alle Disziplinen integrierende Führungsstruktur sichergestellt wurde. Dieses pragmatische Vorgehen führte dazu, dass Lösungen an zufällig sich bietenden Gelegenheiten und nicht an strategischen Notwendigkeiten festgemacht wurden.

\section{Hierarchiedenken versus Aufgabenorientierung}

Mit Begeisterung erzählte ein Arzt von seinen Erfahrungen in einer amerikanischen Klinik, in der sich alle Mitarbeitenden mit der gemeinsamen Aufgabe identifizierten und auf das gemeinsam Geleistete auch sichtbar stolz waren. Genau dies vermisse er in der «Inneren Medizin». 
Die im Grunde einfache Lösung beinhaltete die Etablierung eines «Interdisziplinären Leitungsteams Bettenstation», in dem ein Chefarzt sowie dessen Stellvertreter, die Stationsleitung «Pflege» und die Vertreter(innen) der Administration gemeinsam in regelmässigen Besprechungen über Themen beschliessen, welche die Bettenstation betreffen [2]:

- Implementierung der übergeordneten Standards und Realisierung von konzeptbasierten Massnahmen;

- Sicherstellen einer hochstehenden Qualität in Behandlung und Pflege; auf eine optimale interdisziplinäre Bereitstellung der Ressourcen gewährleisten soll. Sie leistet einen wesentlichen Beitrag zur Behebung der in «Professional Organizations» bestehenden Koordinationsprobleme.

Der Leistungsausweis nach zwei Jahren ist denn auch beeindruckend. Dank koordiniertem interdisziplinärem Vorgehen wurden folgende Vorhaben zum Teil unter Miteinbezug von Ressourcen aus Forschung und Lehre - bearbeitet und die Lösungen sukzessive implementiert und teilweise evaluiert:

- Gemeinsame Patientenvisite auf der Basis eines verbindlichen Standards;

\section{Welche Besprechungen bringen tatsächlich einen Nutzen? Welche Themen gibt es dort? Wer wird dazu eingeladen?}

- Optimierung der gemeinsamen Ressourcen;

- Kontinuierliche Verbesserung der Prozesse und Klärung von wichtigen Schnittstellen;

- Fragen im Zusammenhang der Aus- und Weiterbildung von Ärzten und Pflegenden;

- Laufende Problem- und Konfliktbewältigung bei Eskalationen aus der ausführenden Ebene heraus.

Ergänzend dazu wird auf der Ebene der Klinikleitung in regelmässig abgehaltenen Klausuren die strategische Ausrichtung überprüft und ein allfälliger Umsteuerungsbedarf ermittelt. Die Agenda beinhaltet folgende Themen:

- Positionierung der Klinik im Universitätsspital bzw. im erweiterten Kontext des Gesundheitswesens und Ableitung der passenden Konzepte;

- Entwicklung von Standards und Sicherstellen der Umsetzung der Massnahmen, die aus übergeordneten Konzepten abgeleitet sind;

- Durchsetzung von effizienten Ablauf- und Aufbaustrukturen sowie von Führungssystemen zur Sicherung einer konstanten Qualität;

- Auswahl und Förderung von Schlüsselpersonen.

Das Leitungsgremium überwacht zudem die Umsetzung der gefassten Massnahmen und trifft in regelmässigen Besprechungen Dispositionen zur Koordination von übergreifenden Themen.

Damit wurde, in Ergänzung zur primären Struktur, eine sekundäre Struktur über die Organisation der «Inneren Medizin» gelegt, welche die Gestaltung, Entwicklung und Lenkung der operativen Einheiten den Bettenstationen - sowie des gesamten Bereichs im Hinblick auf eine gemeinsame Ausrichtung und
- Interprofessionelle und interdisziplinäre Patientenprozesse (spitalintern und extern), koordinierte Wahleintritte, patientenfokussierte Ziele und Massnahmen, gemeinsame Durchführung von Untersuchungen, Austrittsplanung;

- Koordination von Anamnese und Eintritts-Screening zwischen Medizin und Pflege (ausgelegt auf den Fokus von chronisch kranken Patienten);

- Konsiliarvisitenregelung zwischen Spezialisten und Generalisten in Medizin und Pflege;

- Implementierung eines Case-Managements;

- Planungsrapport Pflegende;

- Bettenkoordination;

- Einführung eines Critical Incidents Reporting Systems und interprofessioneller Auswertungen;

- Teilweise computerunterstützte Einführungsprogramme für Assistenzärzte und Pflegende.

Alle diese Massnahmen führten letztlich zu einer deutlich gesteigerten Qualität im Kontakt mit dem Patienten. Das fand am deutlichsten bei den Visiten seinen Niederschlag: Durch die durchgehende interprofessionelle Partnerschaft zwischen Ärzten und Pflegenden entwickelte sich sukzessive Exzellenz: zum Wohl des Patienten.

\section{Literatur}

1 Mintzberg H. Mintzberg. On Management - Inside Our Strange World of Organizations. New York: Free Press; 1989.

2 Weber H, Langewitz W. Basler Visitenstandard Chance für eine gelingende Interaktionstriade Patient-Arzt-Pflegefachperson. Psychother Psych Med. 2011;(61):193-5. 